\title{
A systematic review of complications in thoracic spine surgery for ossification of ligamentum flavum
}

\author{
Xiaofei Hou' ${ }^{1}$ Zhongqiang $\mathrm{Chen}^{2} \cdot$ Chuiguo Sun ${ }^{2} \cdot$ Guangwu Zhang ${ }^{1} \cdot$ Sijun $\mathrm{Wu}^{1} \cdot$ Zheng Liu $^{1}$
}

Received: 24 June 2017 / Revised: 12 November 2017 / Accepted: 13 November 2017 / Published online: 28 December 2017

(c) International Spinal Cord Society 2018

\begin{abstract}
Study design Systematic review.

Objectives The aim of this systematic review is to summarize the incidence of complications, to relate complication incidence to procedures performed, to assess the impact of the year of study publication and follow-up duration on complication incidence.

Methods The authors conducted the Cochrane Central Register of Controlled Trials, PubMed, and EMBASE searches for relevant literatures. The incidence of complications was summarized. Correlation of the incidence with year of study publications, follow-up duration, and the surgical outcome was statistically evaluated.

Results A total of 16 studies met our inclusion criteria, including 475 patients. All of these studies were retrospective case series. The mean age of patients ranged from 55 to 64 years. Average follow-up duration ranged from 26 to 65 months. Partial patients in four studies underwent surgeries and reserved posterior structure of the spinal canal. The others underwent operations removing posterior structure of spinal canal. The mean recovery rate from each individual study varied between 31 and $68 \%$ and the pooled neurologic function recovery rate was $53 \%$ (95\% CI: 43-62\%). The mean complication rate was 24\%. Cerebrospinal fluid leakage was the most reported postoperative complication (19\%), then neurologic deterioration (5\%). Other complications included local infections, wound dehiscence, increased kyphotic deformity, an hematoma.

Conclusions Operations removing posterior structure of spinal canal are the main technique to decompress spinal cord. Cerebrospinal fluid leakage and postoperative neurologic deterioration were the most reported complications.
\end{abstract}

\section{Introduction}

Ossification of the ligamentum flavum (OLF), which mainly occurs at the lower thoracic spine, is a potential cause of thoracic spinal canal stenosis and myelopathy [1]. This ectopic ossification has a high prevalence in East Asian populations, particularly in Chinese and Japanese populations $[2,3]$. According to our previous epidemiological study, the standardized prevalence of thoracic OLF is as high as $63.9 \%$ in Chinese people [4].

Once symptoms of thoracic OLF develop, surgical decompression is an effective method with which to relieve

Zhongqiang Chen

puh3_czq@yahoo.com

1 Department of Orthopaedics, Peking University Shougang Hospital, Beijing, China

2 Department of Orthopaedics, Peking University Third Hospital, Beijing, China the spinal cord compression. This surgical procedure is difficult because the caliber of the spinal canal is relatively narrow in the thoracic region, the spinal cord has a tenuous blood supply, and the ossified foci are often adherent to the dura mater [5, 6]. Although extensive studies have investigated surgical methods and clinical outcomes of thoracic OLF surgery during the past few years, the rate of complications remains unclear. The aim of this systematic review is to summarize the incidence of complications of thoracic OLF surgery and relate the incidence of complications to the procedures performed, year of study publication, and follow-up duration.

\section{Methods}

\section{Literature search}

A search for relevant literature was performed using PubMed (www.pubmed.gov), EMBASE (http://www. elsevier.com/online-tools/embase), and the Cochrane 
Central Register of Controlled Trials (CENTRAL) databases. Using the PubMed database as an example, the following search strategy was employed: \#1 search "ossification of ligamentum flavum"; \#2 search [(thoracic) OR thoracic spine] OR thoracic vertebra; \#3 search "thoracic spinal stenosis"; and \#4 search (\#1 and \#2) OR \#3. These terms were modified for use in the other databases as required.

\section{Inclusion criteria}

The following criteria were applied to the studies selected for inclusion in this review: (1) the study contained at least 10 cases of thoracic OLF managed with surgical intervention, (2) the mean follow-up duration was $\geq 1$ year, (3) the study addressed postoperative complications, (4) the patients were $>18$ years of age, and (5) the study was published from July 1974 to October 2015 and was available in full text and in English.

\section{Exclusion criteria}

The exclusion criteria were: (1) studies pertaining to cervical or lumbar OLF and (2) studies involving other spinal surgeries such as cervical or lumbar decompression, anterior decompression of the thoracic spinal canal such as disc herniation, or operations simultaneously addressing ossification of the posterior longitudinal ligament (Fig. 1).

\section{Data collection}

Three reviewers independently extracted the data from the studies selected for inclusion. For each of the eligible studies, we recorded the publication year, patient demographics, follow-up period, surgical approach, preoperative and postoperative neurological assessment scores, recovery rate, and reported complications. When data were missing, the values were excluded from the subsequent analyses.

\section{Statistical analysis}

Demographic data were generated using simple descriptive statistics. Linear regression analysis (SPSS Statistics for Windows version 13.0; SPSS Inc., Chicago, IL, USA) was used to identify any relationships between the incidence of complications and the year of study publication, average age of the patients, follow-up duration or recovery rate. The meta-analysis was performed to analyze the incidence of cerebrospinal fluid leakage (CFL) with Software R 3.4.0. Funnel plot and Egger's test was used to evaluate the publication bias. Statistical significance was defined at a level of $p<0.05$.

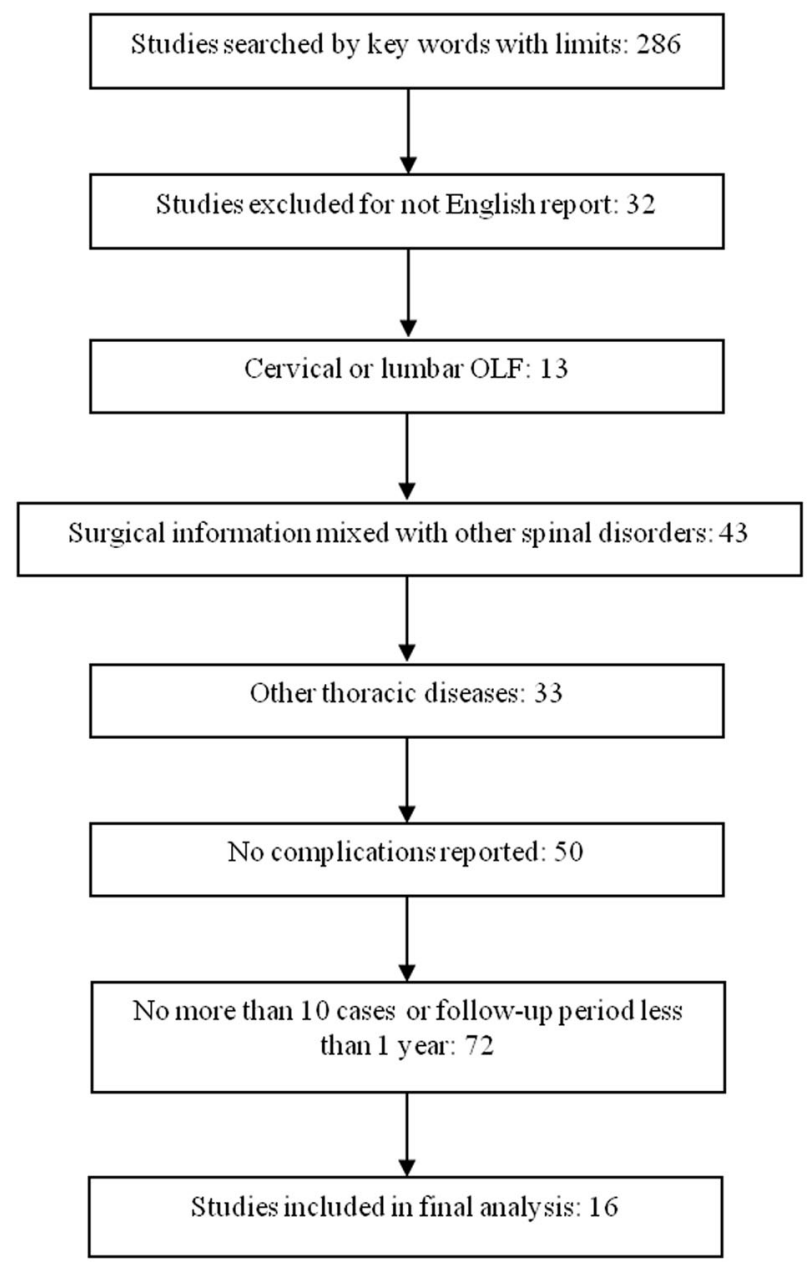

Fig. 1 The flowchart of including or excluding studies according to the criteria and their numbers. OLF ossification of ligamentum flavum

\section{Results}

A total of 16 studies met our inclusion criteria, all of which were retrospective case series. The patient demographics are summarized in Table 1. In total, 475 patients were included in these studies, with a male:female ratio of approximately 1.70:1.00 (297 male and 175 female patients in total, ranging from approximately 4.50:1.00 [17] to 1.00:0.38 [9]). The mean age of the patients ranged from 55 to 64 years, and the average follow-up duration ranged from 26 to 65 months.

The neurological assessment scoring systems used were the modified Japanese Orthopedic Association score, either excluding scores for the upper extremities or just including motor function of the lower extremities. The average preoperative modified Japanese Orthopedic Association score ranged from 3.16 [14] to 6.28 [20]. The mean recovery rate from each individual study varied from 32 [20] to $68 \%$ [13], and the pooled neurological function recovery rate was $53 \%$ (95\% confidence interval: $43-62 \%$ ). 


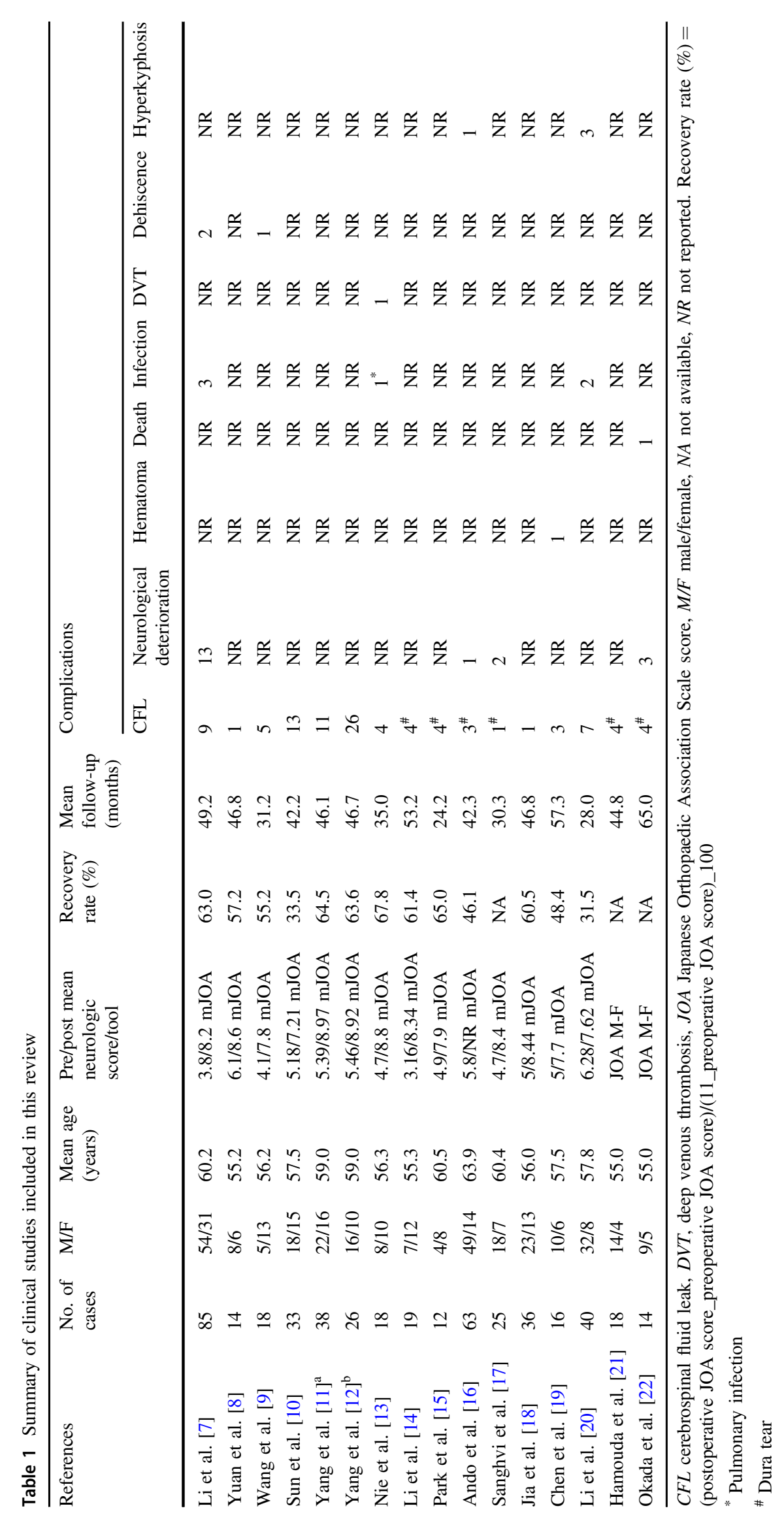




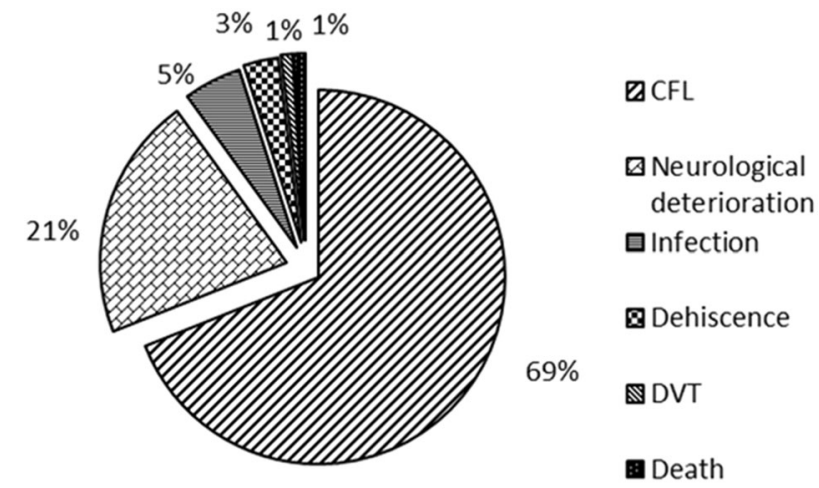

Fig. 2 Proportions of surgical associated postoperative complications of thoracic ossification of ligamentum flavum. The rate means the proximate proportions of the referred complication in total complications. CFL cerebrospinal fluid leak, DVT deep venous thrombosis

In total, 115 complications were reported in the studies (24\% of the 475 patients) (Table 1). The incidence of complications had no statistically significant association with the year of study publication $\left(R^{2}=0.0096, P=0.72\right)$, mean age of the patients $\left(R^{2}=0.0022, P=0.86\right)$, follow-up time $\left(R^{2}=0.0095, P=0.72\right)$, or neurological function recovery $\left(R^{2}=0.0182, P=0.66\right)$. CFL was the most commonly reported postoperative complication, followed by neurological deterioration. Other complications included local infections, wound dehiscence, and others (Fig. 2).

Fifty-four cases of CFL were reported in nine studies (Ref. ${ }^{12}$ was excluded from the meta-analysis due to its high heterogeneity), with an overall postoperative CFL incidence of 19\% (95\% confidence interval: 13-29\%) (Fig. 3a). The $P$-value of Egger linear regression was 0.1973. There was no publication bias (Fig. 3b). Dural defects were operatively repaired using absorbable gelatin sponges, muscle/fascia, or artificial dura. If CFL occurred postoperatively, conservative treatments were employed, such as applying local pressure and positioning the patient in the prone or lateral position. CFL usually ceased within 5-10 days. The major risk factor for CFL was OLF with dural ossification (DO). Two studies addressed the surgical treatment of OLF with DO in this review, and the incidence of CFL in these studies was $39 \%$ [10] and $100 \%$ [12]. The authors did not repair the dural defects but instead meticulously sutured the paraspinous muscles, deep fascia, and skin before placing a subfascial drain and applying continuous pressure to the wound. Pseudocysts were reported in two patients during follow-up [7], and both were cured by ultrasound-guided puncture and aspiration. CFL did not result in any neurological deterioration.

Neurological deterioration was the second most common complication reported in these studies (Fig. 2). In total, 24 patients developed neurological deterioration among five studies involving 227 patients (5\%, based on all patients).
Although postoperative neurological deterioration can be caused by poor operative technique, postoperative hematoma, or local kyphotic deformity, the specific etiology of most cases (18 of 24 cases) was unknown. Most patients were treated with dehydration and corticosteroid therapy, and their neurological deficits were obviously alleviated. However, one patient's deficit persisted for 28 months (until their last reported follow-up). Four patients in two studies $[16,20]$ developed postoperative neurological deterioration due to a local kyphotic deformity. To alleviate their symptoms, two patients underwent additional surgeries and two patients were treated with oral medications. A hematoma occurred in one patient, and the patient's symptoms were alleviated following an emergency operation [19].

Three patients developed wound dehiscence and five patients developed wound infection. These patients improved after irrigation and debridement or with targeted antibiotics. Other complications such as pneumonia and deep vein thrombosis in a lower extremity resolved after appropriate treatment. One patient died of postoperative circulatory and respiratory complications 4 months after surgery.

\section{Discussion}

Decompression is currently the most effective strategy for treating thoracic myelopathy caused by OLF. Although various differentially modified and differentially named surgical procedures have been reported in the literature $[7,8,10,11,13]$, they can be classified as those that either preserve (laminoplasty) or remove (laminectomy) the posterior structures of the spinal canal. Okada et al. [22] compared patients who underwent a laminoplasty procedure with a control group who underwent laminectomy. They found that laminectomy was associated with more complications. However, Li et al. [20] reported four patients who underwent laminoplasty, three of whom had either no change or a worsened neurological status postoperatively. The remaining 36 patients underwent laminectomy, and this procedure yielded better therapeutic effects than laminoplasty. Although some studies have revealed the outcomes of surgeries that preserve the posterior structures of the spinal canal, removal of the posterior wall of the spinal canal remains the main surgical treatment for OLF-related thoracic myelopathy.

In this review, the mean neurological recovery rate varied from $31 \%$ [20] to $68 \%$ [13], generating a pooled neurological function recovery rate of 53\%. Although several recently published articles have reported recovery rates that were obviously lower than the pooled recovery rates among patients with more complicated types of OLF such as OLF with DO and tuberous-like OLF, greater improvement in 
Fig. 3 Forest figure and funnel figure of incidence of postoperative cerebrospinal fluid leakage. a Forest figure of incidence of CFL, random effects model was used to analyzed the data $\left(I^{2}=60.0 \%\right.$, $\left.\operatorname{tau}^{2}=0.3519\right)$. b Funnel plot and Egger linear regression of incidence of CFL. The $P$-value of Egger linear regression was 0.1973 . There was no publication bias
A
Study

Li F 2006

Chen XQ 2009

Jia LS 2010

Nie ZH 2013

Yang Z 2013a

Sun J 2014

Wang T 2014

Yuan Q 2014

Li Z 2015

Fixed effect model

Random effects mode

Heterogeneity: $I^{2}=60 \%, \tau^{2}=0.3519, p<0.01$

Events Total

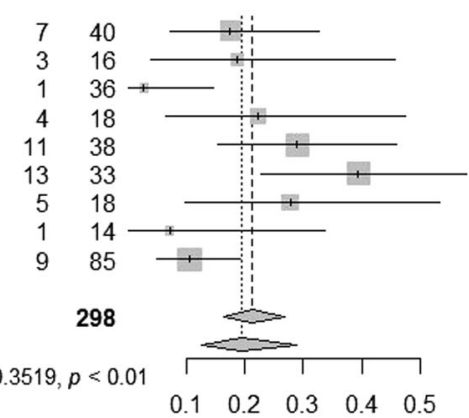

Weight Weight

Proportion $\quad 95 \%-\mathrm{Cl}$ (fixed) (random)

$0.18[0.07 ; 0.33] \quad 14.2 \% \quad 13.6 \%$

$0.19[0.04 ; 0.46] \quad 6.0 \% \quad 9.4 \%$

$0.03[0.00 ; 0.15] \quad 2.4 \% \quad 5.2 \%$

$0.22[0.06 ; 0.48] \quad 7.7 \% \quad 10.6 \%$

$0.29[0.15 ; 0.46] \quad 19.3 \% \quad 14.9 \%$

$0.39[0.23 ; 0.58] \quad 19.4 \% \quad 14.9 \%$

$0.28[0.10 ; 0.53] \quad 8.9 \% \quad 11.4 \%$

$0.07[0.00 ; 0.34] \quad 2.3 \% \quad 5.0 \%$

$0.11[0.05 ; 0.19] \quad 19.8 \% \quad 15.0 \%$

$0.21[0.17 ; 0.27] 100.0 \%$ $0.19[0.13 ; 0.29] \quad-\quad 100.0 \%$

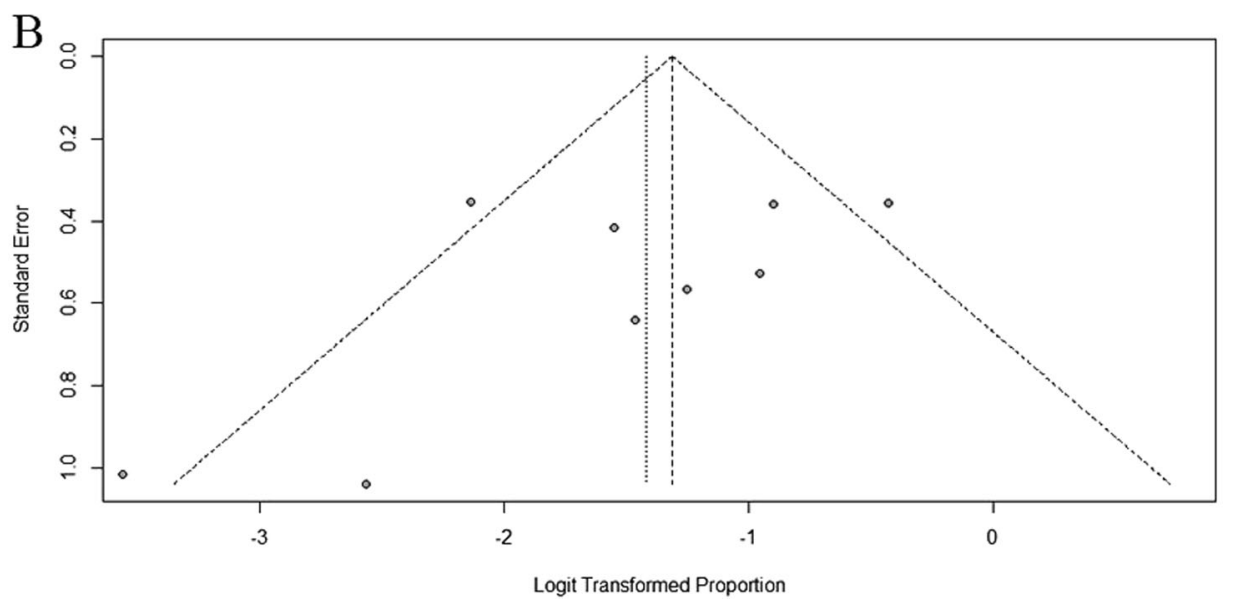

neurological deficits have been observed in most studies published in recent years.

In the present review, the incidence of complications had no statistically significant association with the year of study publication. We speculate that the reason for this finding is that dural tears mostly occurred in the early and mid-term studies in this systematic review. Because the exact number of patients with CFL in these articles remains unknown, the number of patients with dural tears was not counted as the number of patients with CFL. Another reason for the irrelevance between complication rates and variables might be the insufficient sample size of each study (average 30 cases in each study).

CFL is the main complication after thoracic OLF surgery. Inadvertent dural injury during surgery and resection of OLF with DO can lead to CFL. Indeed, DO is a major risk factor for postoperative CFL. Despite the high incidence of CFL after OLF surgery, some studies have suggested that CFL might not influence surgical outcomes [7, 12]. Instead, CFL may increase the difficulty of performing the surgery and postoperative discomfort. Identification of DO in preoperative images could help surgeons to accurately assess the surgical risk. The presence of "tram track" and "comma" signs can be used to predict OLF with
DO on axial computed tomography slices, although the specificity is only $59 \%$ [23].

Another important complication of thoracic OLF surgery is postoperative neurological deterioration. Transient deterioration after surgery is usually associated with injury to the blood supply to the spinal cord and/or improper intraoperative manipulation. Corticosteroids and diuretics should be administered after exclusion of inadequate decompression and a local hematoma. If postoperative neurological deterioration is present and a hematoma is detected, emergency surgery is necessary. Why most patients develop late neurological deterioration remains unclear [7].

In this retrospective study, four patients developed postoperative neurological deterioration because of aggravation of local kyphosis [16, 20]. Whether instrumentation should be used after thoracic OLF decompression surgery is controversial [7]. To reduce the likelihood of postoperative spinal instability or local kyphosis, most surgeons use decompression as well as instrumentation and fusion, especially for conditions such as cervicothoracic or thoracolumbar OLF, multilevel decompression, and decompression involving the majority of facet joints. Yang et al. [12] suggested that use of instrumentation can be avoided if 
spinal instability does not exist preoperatively. However, aging is associated with chronic degenerative changes in the spine, and most patients in these studies underwent thoracic OLF decompression in their 50s and 60s. Therefore, the clinical outcomes of more studies with rigorous designs and long-term follow-up periods are necessary to assess the effect of decompression without the use of instrumentation in the severely degenerated spine.

The incidence of postoperative wound dehiscence, wound infection, pulmonary infection, deep venous thrombosis, and even death is closely associated with many factors, including comorbidities, nutritional status, aging, and the type of operation. Most of these postoperative complications can be managed using approaches such as debridement or administration of targeted antibiotics or anticoagulants. However, careful preoperative evaluation of the patient's general condition and risk factors together with suitable preoperative preparation could decrease the likelihood of these complications arising.

This review has two main limitations. First, all 16 studies examined were retrospective case series, which have inherent weaknesses. However, the use of randomized and prospective observational clinical trials in this field remains controversial. Therefore, systematic retrospective studies are currently the most appropriate method for assessing surgical complications of thoracic OLF. Second, because thoracic OLF mainly occurs in East Asian populations, the exclusion of studies that were published in East Asian languages might have affected our conclusions. However, most of the studies included in this review were carried out by groups in the East Asian region. Therefore, these studies may still accurately represent the status of surgical complications of thoracic OLF.

\section{Data archiving}

There were no data to deposit.

Acknowledgements We acknowledge the statistical advice from Dr. Xiaoxiao Wang (Research Center of Clinical Epidemiology, Peking University Third Hospital). We thank Angela Morben, DVM, ELS, from Liwen Bianji, Edanz Editing China (www.liwenbianji.cn/ac), for editing the English text of a draft of this manuscript. This study was supported by the Beijing Natural Science Foundation (7174366).

\section{Compliance with ethical standards}

Conflict of interest The authors declare that they have no conflict of interest.

\section{References}

1. Hou X, Sun C, Liu X, Liu Z, Qi Q, Guo Z, et al. Clinical features of thoracic spinal stenosis-associated myelopathy: a retrospective analysis of 427 cases. Clin Spine Surg. 2016;29:86-9.
2. Guo JJ, Luk KD, Karppinen J, Yang H, Cheung KM. Prevalence, distribution, and morphology of ossification of the ligamentum flavum: a population study of one thousand seven hundred thirty-six magnetic resonance imaging scans. Spine. 2010;35:51-6.

3. Ohtsuka K, Terayama K, Yanagihara M, Wada K, Kasuga K, Machida T, et al. An epidemiological survey on ossification of ligaments in the cervical and thoracic spine in individuals over 50 years of age. Nihon Seikeigeka Gakkai zasshi. 1986;60: 1087-98.

4. Lang N, Yuan HS, Wang HL, Liao J, Li M, Guo FX, et al. Epidemiological survey of ossification of the ligamentum flavum in thoracic spine: CT imaging observation of 993 cases. Eur Spine J. 2013;22:857-62.

5. Lazorthes G, Gouaze A, Zadeh JO, Santini JJ, Lazorthes Y, Burdin P, et al. Arterial vascularization of the spinal cord. Recent studies of the anastomotic substitution pathways. J Neurosurg. 1971;35:253-62.

6. Barnett GH, Hardy RW Jr, Little JR, Bay JW, Sypert GW. Thoracic spinal canal stenosis. J Neurosurg. 1987;66:338-44.

7. Li Z, Ren D, Zhao Y, Hou S, Li L, Yu S, et al. Clinical characteristics and surgical outcome of thoracic myelopathy caused by ossification of the ligamentum flavum: a retrospective analysis of 85 cases. Spinal Cord. 2016;54:188-96.

8. Yuan Q, Zheng S, Tian W. Computer-assisted minimally invasive spine surgery for resection of ossification of the ligamentum flavum in the thoracic spine. Chin Med J (Engl). 2014;127:2043-7.

9. Wang T, Yin C, Wang D, Li S, Chen X. Surgical technique for decompression of severe thoracic myelopathy due to tuberous ossification of Ligamentum flavum. Clin Spine Surg. 2017;30: E7-12.

10. Sun J, Zhang C, Ning G, Li Y, Li Y, Wang P, et al. Surgical strategies for ossified ligamentum flavum associated with dural ossification in thoracic spinal stenosis. $\mathbf{J}$ Clin Neurosci. 2014;21:2102-6.

11. Yang Z, Xue Y, Dai Q, Zhang C, Zhou HF, Pan JF, et al. Upper facet joint en bloc resection for the treatment of thoracic myelopathy caused by ossification of the ligamentum flavum. J Neurosurg Spine. 2013;19:81-9.

12. Yang Z, Xue Y, Zhang C, Dai Q, Zhou H. Surgical treatment of ossification of the ligamentum flavum associated with dural ossification in the thoracic spine. J Clin Neurosci. 2013;20:212-6.

13. Nie ZH, Liu FJ, Shen Y, Ding WY, Wang LF. Lamina osteotomy and replantation with miniplate fixation for thoracic myelopathy due to ossification of the ligamentum flavum. Orthopedics. 2013;36:e353-9.

14. Li M, Wang Z, Du J, Luo Z, Wang Z. Thoracic myelopathy caused by ossification of the ligamentum flavum: a retrospective study in Chinese patients. J Spinal Disord Tech. 2013;26: E35-40.

15. Park DA, Kim SW, Lee SM, Kim CG, Jang SJ, Ju CI. Symptomatic myelopathy caused by ossification of the yellow ligament. Korean J Spine. 2012;9:348-51.

16. Ando K, Imagama S, Wakao N, Hirano K, Tauchi R, Muramoto A, et al. Examination of the influence of ossification of the anterior longitudinal ligament on symptom progression and surgical outcome of ossification of the thoracic ligamentum flavum: a multicenter study. J Neurosurg Spine. 2012;16:147-53.

17. Sanghvi AV, Chhabra HS, Mascarenhas AA, Mittal VK, Sangondimath GM. Thoracic myelopathy due to ossification of ligamentum flavum: a retrospective analysis of predictors of surgical outcome and factors affecting preoperative neurological status. Eur Spine J. 2011;20:205-15.

18. Jia LS, Chen XS, Zhou SY, Shao J, Zhu W. En bloc resection of lamina and ossified ligamentum flavum in the treatment of 
thoracic ossification of the ligamentum flavum. Neurosurgery. 2010;66:1181-6.

19. Chen XQ, Yang HL, Wang GL, Gu Y, Pan WM, Dong RB, et al. Surgery for thoracic myelopathy caused by ossification of the ligamentum flavum. J Clin Neurosci. 2009;16:1316-20.

20. Li F, Chen Q, Xu K. Surgical treatment of 40 patients with thoracic ossification of the ligamentum flavum. J Neurosurg Spine. 2006;4:191-7.
21. Ben Hamouda K, Jemel H, Haouet S, Khaldi M. Thoracic myelopathy caused by ossification of the ligamentum flavum: a report of 18 cases. J Neurosurg. 2003;99:157-61.

22. Okada K, Oka S, Tohge K, Ono K, Yonenobu K, Hosoya T. Thoracic myelopathy caused by ossification of the ligamentum flavum. Clin Study Surg Treat Spine. 1991;16:280-7.

23. Muthukumar N. Dural ossification in ossification of the ligamentum flavum: a preliminary report. Spine. 2009;34:2654-61. 